\title{
Use of Acoustic Energy in Sand Casting of Aluminium Alloys PUGA H. ${ }^{1, a}$, BARBOSA J., b, OLIVEIRA J.1,c \\ ${ }^{1}$ CT2M - Centre for Mechanical and Materials Technologies \\ Universidade do Minho, 4800-058 Guimarães, Portugal

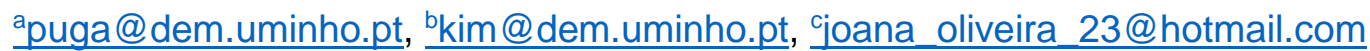

Keywords: Ultrasonic, Casting, Aluminium Alloy

\begin{abstract}
During the last years, some researchers have focused the development of ultrasonic microstructure refinement /modification techniques of die-casting aluminium alloys, to improve their properties. The developed techniques are highly efficient when applied to the die-casting process, but their capability with sand and ceramic moulding are unknown.

Sand/ceramic aluminium castings are prone to coarse microstructure, porosities and inclusions due to low cooling rates and turbulent gravity pouring, and suitable processing techniques are required to eliminate those drawbacks. This article reports some results of a research work aiming the development of a reliable, low-cost and environmentally friend casting process, for geometrically complex and massive high strength sand/ceramic aluminium castings, to eliminate traditional soundness related defects and simultaneously promote the development of refined microstructures. The article presents the effect of applying ultrasound to $\mathrm{AlSi}_{9} \mathrm{Cu}_{3}$ alloy during solidification on a sand mould on the resultant microstructure. Results include microstructure characterization and its relationship with thermal analysis data collected from the center of the cast samples during cooling.
\end{abstract}

\section{Introduction}

Al-Si based alloys are widely used in massive and large components where high strength-to-weight ratio is required. Although certain geometries can be produced by die-casting, medium and large castings are usually obtained by sand casting, which is very prone to coarse microstructure and inclusions, due to low cooling rates, erosion of the mould walls and turbulent filling [1].

Microstructure usually consists of coarse dendritic - $\mathrm{Al}$ solid solution phase, acicular eutectic silicon and intermetallic phases, which are highly detrimental to tensile and fatigue properties [2]. Fatigue is the most important mechanism associated to $\mathrm{Al}$ components failure in service and, besides microstructure, it is extremely sensitive to casting defects, mainly porosities and inclusions [3]. Castings with defects show at least an order of magnitude lower fatigue life compared to defect free castings [4].Thus, casting defects control and efficient microstructure refinement/modification are imperative to achieve high mechanical performance.

The major problem associated to aluminium castings is the inability to completely eliminate production defects and simultaneously obtain fully refined microstructures to enhance components performance. Chemical refinement doesn't avoid dendritic structures, has no effect over intermetallic phases and is not able to decrease grain size to values below $200 \mu \mathrm{m} \mathrm{[5,6].} \mathrm{Si}$ modification is crucial to achieve high mechanical properties [7], but current chemical modification techniques increase porosity [8,9] and generate great amount of dross and smoke [10].

Ultrasonic refinement/modification is a valuable alternative to traditional chemical techniques and overtake their associated problems. When a liquid metal is submitted to high intensity ultrasonic vibrations, the alternating pressure above the cavitation threshold brings about numerous tiny bubbles in the liquid metal, which start growing, pulsing and finally collapse. During expansion, bubbles absorb energy from the melt, undercooling the liquid at the bubble-liquid interface, resulting in nucleation at the bubble surface [11]. When bubbles collapse, acoustic streaming develops in the melt, distributing the nuclei into the surrounding liquid, thus promoting heterogeneous nucleation. If the melt temperature is low enough to avoid dissolution of the nuclei, embryos life time is long enough to promote the development of large number of globular grains 
with grain size less than $40 \mu \mathrm{m}$ [12]. Besides grain refinement, cavitation was found to refine intermetallic phases and distribute them uniformly throughout the matrix and modify eutectic silicon lamellae into fibrous particles with less than $2 \mu$ m length [13].

\section{Experimental Procedure}

Experimental set-up. Fig. 1 shows the experimental set-up used to perform ultrasonic grain refinement/modification. It consists of a novel MMM (Multi-frequency, Multimode, Modulated technology) US power supply unit developed by MP Interconsulting, a high power ultrasonic converter $(1200 \mathrm{~W})$, a $15 \mathrm{~mm}$ diameter and $150 \mathrm{~mm}$ long acoustic waveguide made of $\mathrm{Ti}_{6} \mathrm{Al}_{4} \mathrm{~V}$ and the mould itself. Dimensions of cast sample were $70 \times 50 \times 20 \mathrm{~mm}$.

The $\mathrm{AlSi}_{9} \mathrm{Cu}_{3}$ was melted and held inside the crucible at $700{ }^{\circ} \mathrm{C}$ during 30 minutes for homogenization. The molten alloy was then allowed to cool until $680{ }^{\circ} \mathrm{C}$ and poured in the sand casting mould. For every experimental condition, US vibration was applied continually between 680 and $580{ }^{\circ} \mathrm{C}$ melt temperatures, after which the US unit was stopped, and the melt cooled until room temperature. Melt temperature was controlled within an accuracy of $\pm 2{ }^{\circ} \mathrm{C}$. For the sake of comparison experiments were also carried out without ultrasonic vibration. US vibration was performed using $400 \mathrm{~W}$ electric power and $19.8 \mathrm{kHz}$ frequency using $\pm 0.25 \mathrm{kHz}$ sweeping. For online motorization and acquisition of metal temperature a LabVIEW based dedicated program was used together with a CompactDAQ (cDAQ) National Instruments acquisition system.

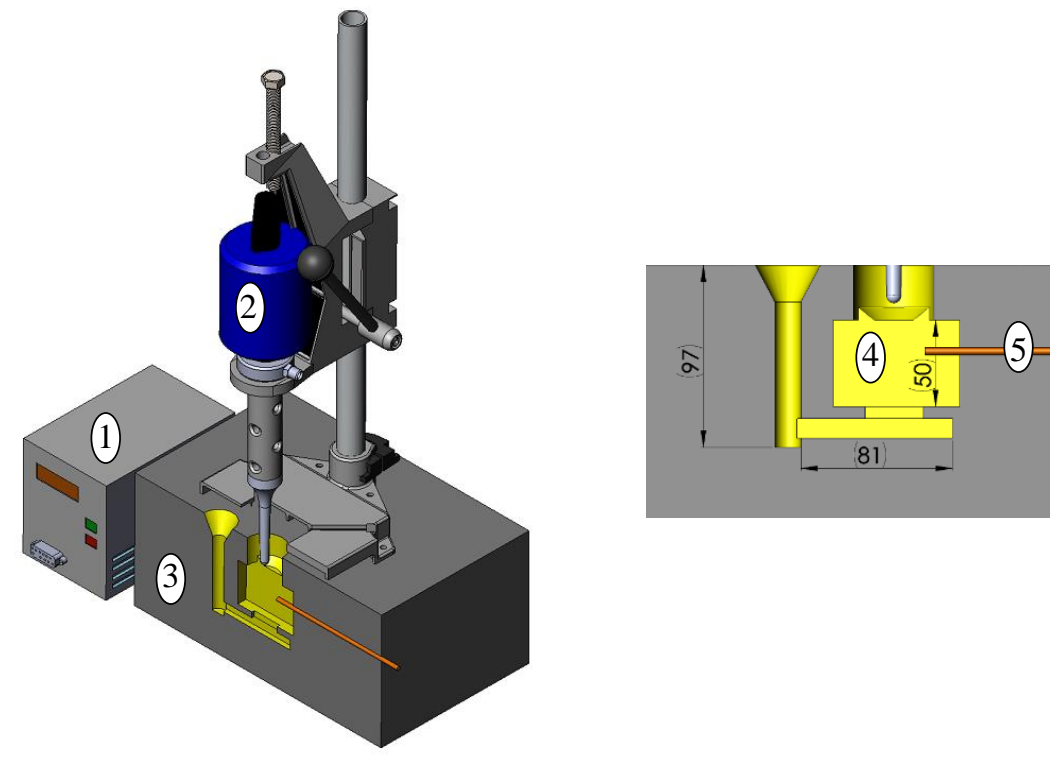

Fig.1. Experimental set-up - Conceptual model: 1- US supply unit, 2 - US converter, 3 - Sand Mould, 4 - Casting, 5 Thermocouple.

Samples characterization. Samples for microstructure characterization were taken from each cast sample by sectioning and ground using $1200 \mathrm{SiC}$ paper and polished up to $1 \mu \mathrm{m}$. Those samples used for optical microscopy characterization were etched using Keller's reagent to reveal the resulting microstructure. Optical microscope (OM) and Scanning Electron Microscope (SEM) with quantitative metallographic analysis capability were used to evaluate shape and grain size of constituents.

\section{Experimental results and discussion}

Fig. 2 (a) shows an optical micrograph of the microstructure of $\mathrm{AlSi}_{9} \mathrm{Cu}_{3}$ as-cast sample obtained without supplying acoustic energy. The microstructure exhibits fully developed primary $\alpha$-Al 
dendrites and the traditional coarse acicular eutectic Si. Besides $\alpha$-Al and silicon plates, SEM analysis revealed the co-existence of other intermetallic phases, namely the long and sharp needles of $\beta-\mathrm{Al}_{5} \mathrm{FeSi}$ as presented in Fig. 2 (b) which are known to be highly detrimental to the mechanical properties of the alloy, particularly ductility [14].
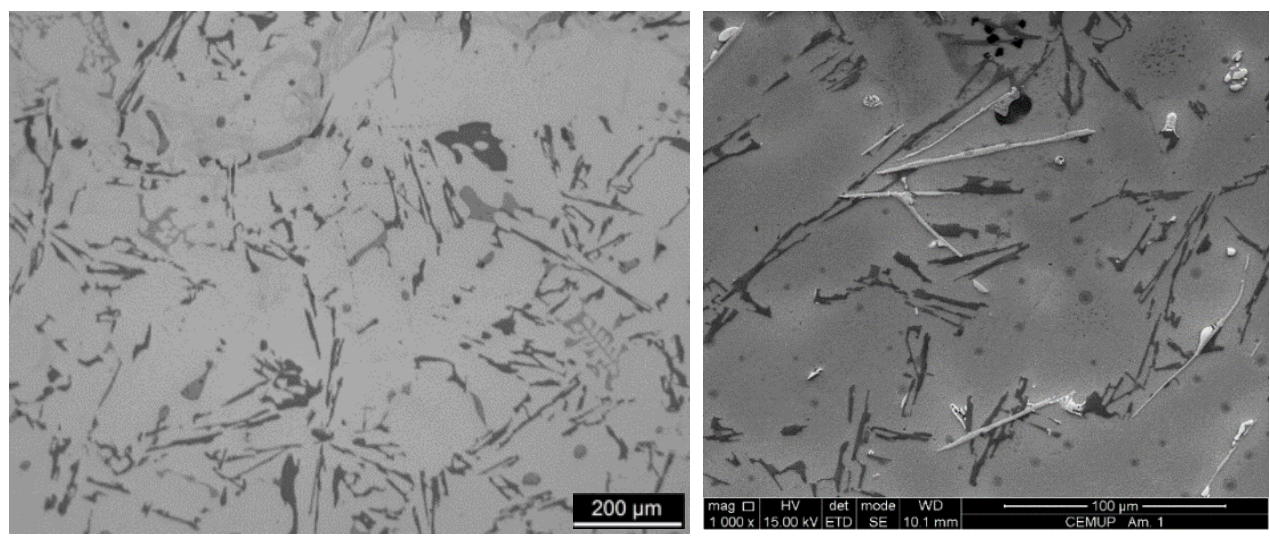

Fig. 2 Microstructure of as-cast $\mathrm{AlSi}_{9} \mathrm{Cu}_{3}$ without treatment with acoustic energy. (a) Optical image; (b) SEM image.

Fig. 3 (a) presents the as-cast microstructure of the AlSi9Cu3 alloy submitted to acoustic energy. It is clear that ultrasonic treatment had a strong effect on the microstructure morphology of the as-cast alloy. US treatment promoted the formation of a mixture of globular and coarse rosette like $\alpha$-Al grains with average size around $150 \mu \mathrm{m}$. Moreover, Figs. 3 (a) and (b) also suggest that size, thickness and spacing between eutectic silicon lamellae trend to decrease with ultrasonic treatment. SEM characterization (Fig.3 (b)) suggest the presence of well distributed intermetallic phases $\alpha$ $\mathrm{Al}_{15}(\mathrm{Mn}, \mathrm{Fe})_{3} \mathrm{Si}_{2}$ and $\beta-\mathrm{Al}_{5} \mathrm{FeSi}$ with branch arms shorter than those detected in the microstructure of non US treated samples (Fig. 2 (b)) that may certainly have a positive effect in mechanical properties, as it has already been observed for die-cast US treated samples [13].
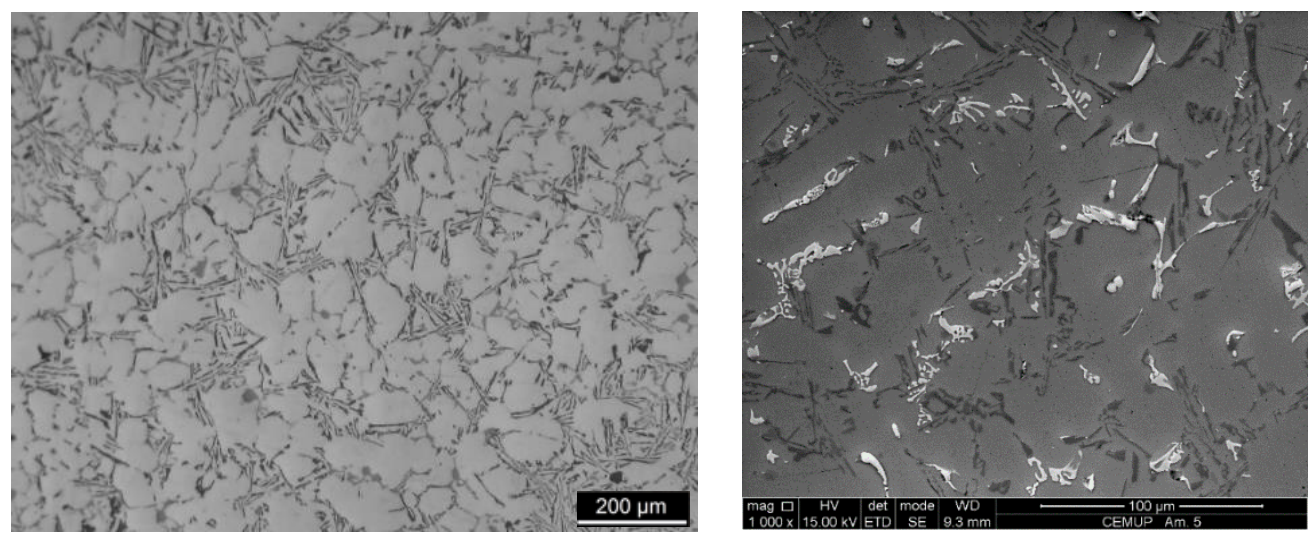

Fig. 3 Microstructure of as-cast AlSi9Cu3 with treatment by acoustic energy. (a) Optical image; (b) SEM image.

Fig. 4 shows the cooling curves obtained for samples without and with US treatment during solidification. Precipitation of proeutectic $\alpha$-Al starts at higher temperature in the sample submitted to US when compared to that solidified without US supply. This is in agreement with the refinement effect on this phase observed by comparing microstructures of Fig. 2 (a) and Fig 3 (a). In what concerns to the eutectic reaction of the sample processed by ultrasound, it also starts at higher temperature and has a duration time shorter than that observed for the non US treated sample. The differences detected in solidification curves and the resultant microstructures, clearly suggest that US supply worked as germinator of solidification nuclei both on the beginning of proeutectic precipitation and the beginning of the eutectic reaction. 


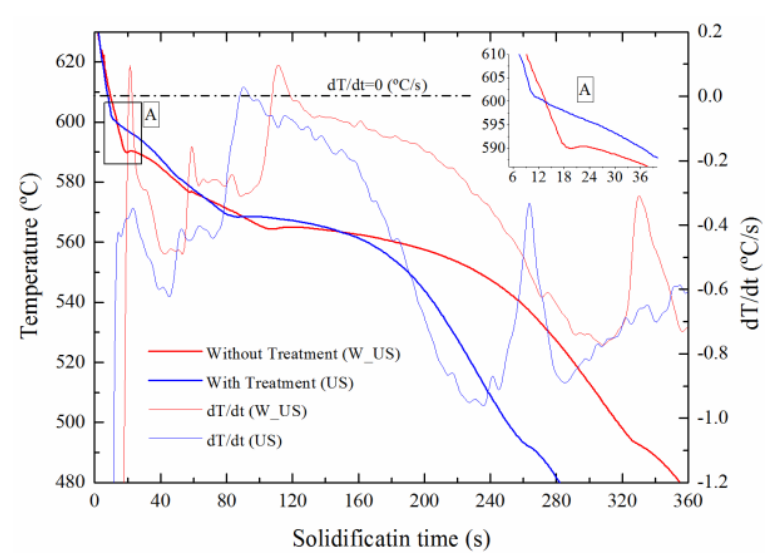

Fig. 4 Thermal analysis data collected from a thermocouple in the center of sample during solidification.

\section{Summary}

Results show that ultrasound promotes refinement of the $\alpha$-Al and eutectic Si phases, reduces the size and morphology of intermetallic phases and distribute them uniformly in the matrix. For the processing conditions, namely US parameters, ultrasonic processing promotes the change of traditional $\alpha$-Al dendritic morphology of sand castings into a mix of globular and rosette-like morphology with average grain size around $150 \mu \mathrm{m}$. Although it was not quantified, it is also evident that US decreases size, thickness and spacing between eutectic silicon lamellae and the size and morphology of intermetallic phases. Changes observed in the microstructure are confirmed by changes in the cooling curves of the US treated samples, namely an increase in the starting temperatures of solidification and the eutectic reaction, as well as a decrease in eutectic reaction duration time.

\section{Acknowledgements}

This research was supported by FCT - Portuguese Foundation for Science and Technology and was developed on the aim of the research project PTDC/EME-TME/119658/2010 and the Post-Doctoral grant SFRH/BPD/76680/2011.

\section{References}

[1] X. Day, X. Yang, J. Campbell, J. Wood: Mater. Sci. Eng. A 354 (2003), p. 315- 325

[2] K.G. Basavakumar, P.G. Mukunda, M. Chakraborty: Mater. Character. 59 (2008), p. 283-289

[3] H.R. Ammar, A.M. Samuel, F.H. Samuel: Mater. Sci. Eng. A 473 (2008), p. $65-75$

[4] Q.G. Wang, D. Apelian, D.A. Lados: J. Light Met. (2001), p. 73-84

[5] G.K. Sigworth, T.A. Kuhn: AFS Tran. 67 (2) (2007), p. 1-12

[6] J.A. Spittle: Int. J. Cast. Metal. Res. 19 (2006), p. 210-222

[7] J.A. García-Hinojosa, C.R. González, G.M. González, Y. Houbaert: J Mater Process Tech 143144 (2003), p. 306-310

[8] S.M. Miresmaeili, J. Campbell, S.G. Shabestari, S.M.A. Boutorabi: Metall. Mater. Tran. A 36A (2005), p. 2342-2349

[9] S.D. Mcdonald, A.K. Dahle, J.A. Taylor, D.H. StJohn: Metall. Mater. Tran. B 35B (2004), p. 1097-1106

[10] H. Puga, J. Barbosa, D. Soares, F. Silva, S. Ribeiro: J. Mater. Process. Tech. 209 (2009) 51955203

[11] G.I. Eskin in: Ultrasonic Treatment of Light Alloy Melts, edited by Gordon and Breach Science Publishers, Amsterdam (1998).

[12] W. Khalifa, Y. Tsunekawa, M. Okumiya: Int. J. Cast. Metal. Res.21 (2008), p. 129-134.

[13] H. Puga, J. Barbosa, S. Costa, S. Ribeiro, A.M.P. Pinto, M. Prokic: Mater. Sci. Eng. A 560 (2013), p. 589-595

[14] E. Tillova, M. Panuskova: Metalurgija 47 (2008), p. 207-210 